\title{
Iterative Solvers for Dynamically Implicit Reservoir Flow Equations on Irregular Grids
}

\author{
BRAND, Clemens W., and GANZER, Leonhard, Montanuniversität Leoben, Austria
}

Paper presented at the 5th European Conference on the Mathematics of Oil Recovery, Leoben, Austria, 3-6 Sept. 1996

\section{ABSTRACT}

Modern reservoir simulators allow for local refinement and other types of irregular grids. Moreover, the number and type of flow equations will not be the same in all blocks. Depending on the state of the reservoir, some parts may employ a fully implicit discretization, others an IMPES formulation; some parts will use a compositional model and others will just have one-phase flow. These features, while giving flexibility to the reservoir engineer, produce large systems of linear equations with highly irregular structure. Depending on the flow model, the number of gridblocks, the number of implicitly discretized variables per gridblock and the relative importance of pressure terms versus concentration derivatives, these equations can consume excessive amounts of computer resources. We describe iterative solvers that work (almost) as blackbox routines within a commercial reservoir simulator. The solvers use incomplete $L U$-factorization to precondition restarted ORTHOMIN iterations. Memory constraints forbid to store many orthogonal vectors. Thus preconditioning has to be efficient. Our program adjusts the amount of fill in the preconditioner depending on the "difficulty" of the problem. The symbolic factorization is based on the block system (which does not change), and not on the actual matrix structure (which changes whenever the implicitness or the number of equations in a block changes). This trick minimizes calls to the (time-consuming) symbolic factorization part while still allowing changes in matrix structure. Another important feature is to treat fill-in terms differently depending on the type of unknown. For pressure terms, we keep fill-in to a higher degree than for saturation or concentration.

\section{INTRODUCTION}

The systems of linear equations that result from discretization and linearization of reservoir flow equations are a tough challenge for iterative solvers. They need powerful preconditioners to converge in a reasonable number of iterations. A broad class of preconditioners is based on incomplete factorization of the coefficient matrix. Many standard sources, e. g. [3, 6, 8], describe how to construct and implement preconditioners of type $\mathrm{I} L U(0)$, that is, with no additional fill terms. Preconditioners that allow higher degree of fill are usually described for banded matrices only.

In this paper we describe the implementation of preconditioners with considerable fill and arbitrary sparsity pattern, and we discuss the reasons for using this type of preconditioning. The first section explains the demands that a solver for dynamically implicit reservoir flow equations has to meet, and we justify the choice of high-degree incomplete factorization. The rest of the paper deals with algorithms and their implementations.

\section{THE SOLVER WITHIN A SIMULATOR}

Software engineering teaches to encapsulate the various tasks of a program into separate modules. The less one module needs to know about the other, the better. In this way of thinking, the simulator provides a set of equations and hands the linear system to the solver. The solver finds the solution and sends it back to the simulator. Ideally, the interaction between these two may be restricted to the exchange of a matrix, a right-hand side and a solution. However, this blackbox approach is too simplistic.

It is not possible to isolate the solver from the rest of the simulator. (We talk about iterative solvers; for direct solvers the situation is different!) Theoret- 
ically, matrix and right-hand side of a linear system contain all the information needed to solve the equations. However, for an iterative solver some systems are more difficult to solve than others of the same size. The simulator, which controls parameters like length of time-step or degree of implicitness, should set up linear equations so that the linear solver can complete its task properly. Generally, the more that is known about the interaction between the reservoir model and the solver, the more the algorithms can be tuned.

The important criterion for efficiency is not: how fast can a linear solver solve one system, but: how fast are simulator and solver together to complete $a$ simulation.

Elements of Interaction. Solver and simulator interact in more ways than just by exchanging matrix and solution. The diagram in Figure 1 shows within the structure of a reservoir simulator those elements that have a bearing on the linear solver. In the following, we will address these elements in detail.

\begin{tabular}{|c|}
\hline $\begin{array}{c}\text { grid construction } \\
\text { regular vs. irregular structure of matrix } \\
\text { model equations } \\
\text { same flow model in all blocks, or not } \leftrightarrow \\
\text { same number and type of equations } \\
\text { in all blocks, or not }\end{array}$ \\
\hline $\begin{array}{c}\text { do for all time steps } \\
\text { select time step } \\
\text { steps short or long } \leftrightarrow \\
\text { linear system easy or hard to solve }\end{array}$ \\
$\begin{array}{r}\text { do for all nonlinear iterations } \\
\text { adaptive-implicit formulation } \\
\text { determines size of linear system }\end{array}$ \\
$\begin{array}{c}\text { accuracy threshold for inner iterations } \\
\text { influences outer and inner iterations } \\
\text { Linear solver } \\
\text { construct preconditioner } \\
\text { simple vs. elaborate }\end{array}$ \\
\hline $\begin{array}{r}\text { do linear iterations } \\
\text { acceleration scheme } \\
\text { store and orthogonalize } \\
\text { more or less orthonormal vectors } \\
\text { update solution }\end{array}$ \\
\hline
\end{tabular}

FIGURE 1: Interaction between simulator and solver

Regular Versus Irregular Structure. For modeling a reservoir, two alternatives have been pursued. One approach is: Keep grid and computational model simple and regular! Use a cartesian $I \times J \times K$ grid. Have the same number of phases and components in all blocks. Set up either an IMPES model or a fully implicit model, but do not mix. (The geometric shape of the gridblocks may be distorted to match the struc- tures in the reservoir; but as far as data structures are concerned, the grid remains a cartesian product.) The simple data structure allows to implement highly efficient algorithms.

The other approach is: Allow great freedom in gridding and modeling! Refine gridblocks, let them have irregular connections and shapes for modeling faults, boundaries and the vicinity of wells. Use an Impes discretization for most of the reservoir and a fully implicit formulation in few blocks only. Allow blocks of different type (water, black-oil, compositional...). The price for this flexibility is high: Data structures are complicated. The linear solver encounters equations with irregular structure.

Certainly, both approaches are justified for different types of problems. Here we just want to illustrate two points: The linear solver must fit the simulator; and the solver's efficiency can be judged only by the performance of the entire simulator.

Inner and Outer Iterations. Reservoir flow equations (unless greatly simplified) are nonlinear. Newton's method solves nonlinear equations iteratively. At each step it computes the residual and the Jacobian matrix. It then solves the associated linear system, usually again by an iterative method. Thus, there are inner, or linear, iterations nested within a loop of outer, or nonlinear, iterations.

Two ideas will help saving work in the inner iterations at the expense of possibly increasing the number of outer iterations. First, the iterative linear solver may set a moderately accurate stopping criterion, as long as the outer iterations are not yet completed. Since each step of Newton's method computes just another correction to the approximate solution, there is no need to calculate these corrections with undue accuracy. Second, we may work with a simplified approximation to the original Jacobian matrix, so that most of the variables become decoupled from the system. An adaptive-implicit formulation achieves exactly that.

Fully implicit discretization provides stable solutions and allows larger time steps than an IMPES approach-but it involves much more unknowns and linear systems that are harder to solve by iterative methods. Thomas and Thurnau[13] introduced an adaptive-implicit formulation. There, the number of variables is only moderately higher than in a purely IMPES formulation. When the explicit variables are updated after each step, the method is essentially Newton's method with an inexact Jacobian matrix. Consequently, it still converges to a fully implicit solution.

Thus, for good total performance the simulator carefully has to choose which variables it should treat implicitly or explicitly; it also has to specify appropriate accuracy thresholds for the inner iterations. However, the rules set up in our simulator are mostly empirical, 
based on trial and error.

Length of Time Step. There is another parameter with profound implications for the linear solver's speed of convergence: the length of the time step. The smaller the time step, the faster both the outer, nonlinear iterations of Newton's method and the inner iterations of the linear solver will converge (usually). Of course, then more steps are necessary to complete a simulation. The routine that controls the time step contributes a good deal to the total speed of a simulator. An algorithm that could predict a priori a relation between the length of the time step and the convergence properties of the associated linear system would be highly desirable. Unfortunately, again there are no straight and simple rules.

Preconditioning-Iteration-Acceleration. So far, we have discussed how formulation, discretization and linearization of a flow model-issues that the linear solver cannot control directly-may affect the linear solver and the total performance of the simulator. Now, let us look at those parameters that the solver can control.

Computational work and demands on memory within an iterative solver are typically distributed between three tasks: construction of a preconditioner; iterative update of solution and residual; implementation of some nonstationary acceleration scheme.

One extreme example would be the plain Jacobi method. It invests nothing into preconditioning or acceleration, and thus needs a prohibitively high number of iterations to solve all but the most simple problems. On the other hand, consider Vinsome's ORTHOMIN[14], which is a usual acceleration method in reservoir simulators. The more vectors it keeps in its orthonormal basis, the faster it converges. Thus, at the cost of more memory for storing the basis vectors, and more computational work for orthogonalization, ORTHOMIN may compensate for a weak preconditioner. Finally, as another extreme example, preconditioning by an exact factorization of the matrix would solve any linear system in just one iteration. This, however, is equivalent in terms of work and storage to a direct solution, which is unfeasible for large systems.

A reasonable compromise for an efficient solver, as we propose it, will invest in the construction of a good preconditioner arithmetic work equivalent to one or two iterations. It will store a moderate number of basis vectors ${ }^{1}$ for ORTHOMIN. Ideally, it should converge then in about twenty or thirty iterations. Still, we have to repeat what was said above: these rules are based much more on experience than on firm theory.

\footnotetext{
${ }^{1}$ Our default value is 5 . Other simulators recommend higher values. The reason for this seems that we devote more work to preconditioning
}

\section{INCOMPLETE FACTORIZATION}

A matrix that stems from an adaptive-implicit formulation of flow equations on an irregular grid has a highly irregular sparsity pattern. Neither is it symmetric nor diagonally dominant. Iterative solvers need powerful preconditioning to reach reasonable rates of convergence for these systems. Preconditioners based on incomplete factorization are well suited for this task.

Meijerink and Van der Vorst[10] first investigated incomplete $L U$-decompositions of symmetric $M$ matrices. (A matrix $A=\left(a_{i, j}\right)$ is an $M$-matrix if it is nonsingular, $a_{i, j} \leq 0$ for $i \neq j$ and all entries in $A^{-1}$ are nonnegative. For example, in an IMPES model the linear system for the block pressures has this property.)

An incomplete factorization is constructed by $L U$ decomposition similar to Gaussian elimination. During the factorization certain fill elements, in positions that are zero in the original matrix but would become nonzero in an exact factorization, are ignored. A class of preconditioners with varying efficacy can be obtained by adjusting the number of fill elements. In the limit, when the factorization retains all fill terms, the iterative method even becomes a direct solver. An incomplete factorization that retains higher-order fill elements, however, needs a considerable amount of computational work. Those parts of the algorithm that do most of the work are highly sequential. Therefore, incomplete factorization with higher-order fill elements is not suited for parallel computers. On vector computers or workstations, however, the algorithm shows its full strength. Considerations for implementing $I L U$ preconditioners on vector and shared memory computers are given in reference [6], but unfortunately, few of the methods presented there apply to high-degree I $L U$-factorizations.

An overview on other preconditioners, such as Jacobi, SSOR, ADI or polynomial preconditioners, is given, for example, by Barret et al.[3]. Appleyard and Cheshire[1] introduced nested factorization, a preconditioner designed specifically for three-dimensional $I \times J \times K$-type grids. Compared to these methods, incomplete factorization has two advantages: it does not depend on a certain type of grid or a specific numbering of gridblocks; and it is possible to tune its efficacy by selecting the amount of fill.

Fill levels. Our implementation of incomplete factorization retains or discards fill terms according to their level. An entry in a position where the original matrix has a nonzero entry is assigned level zero. The factorization procedure updates entry $a_{i, j}$ by the operation $a_{i, j} \leftarrow a_{i, j}-a_{i, k} a_{k, j} / a_{k, k}$. When $a_{i, k} a_{k, j} / a_{k, k} \neq 0$, fill occurs. The level of this fill term is the sum of the levels of $a_{i, k}$ and $a_{k, j}$ plus one.

Definition. A factorization that retains fill terms up 
Algorithm 1 The incomplete factorization $I L U(\ell)$, where the level of fill terms is updated during the factorizations.

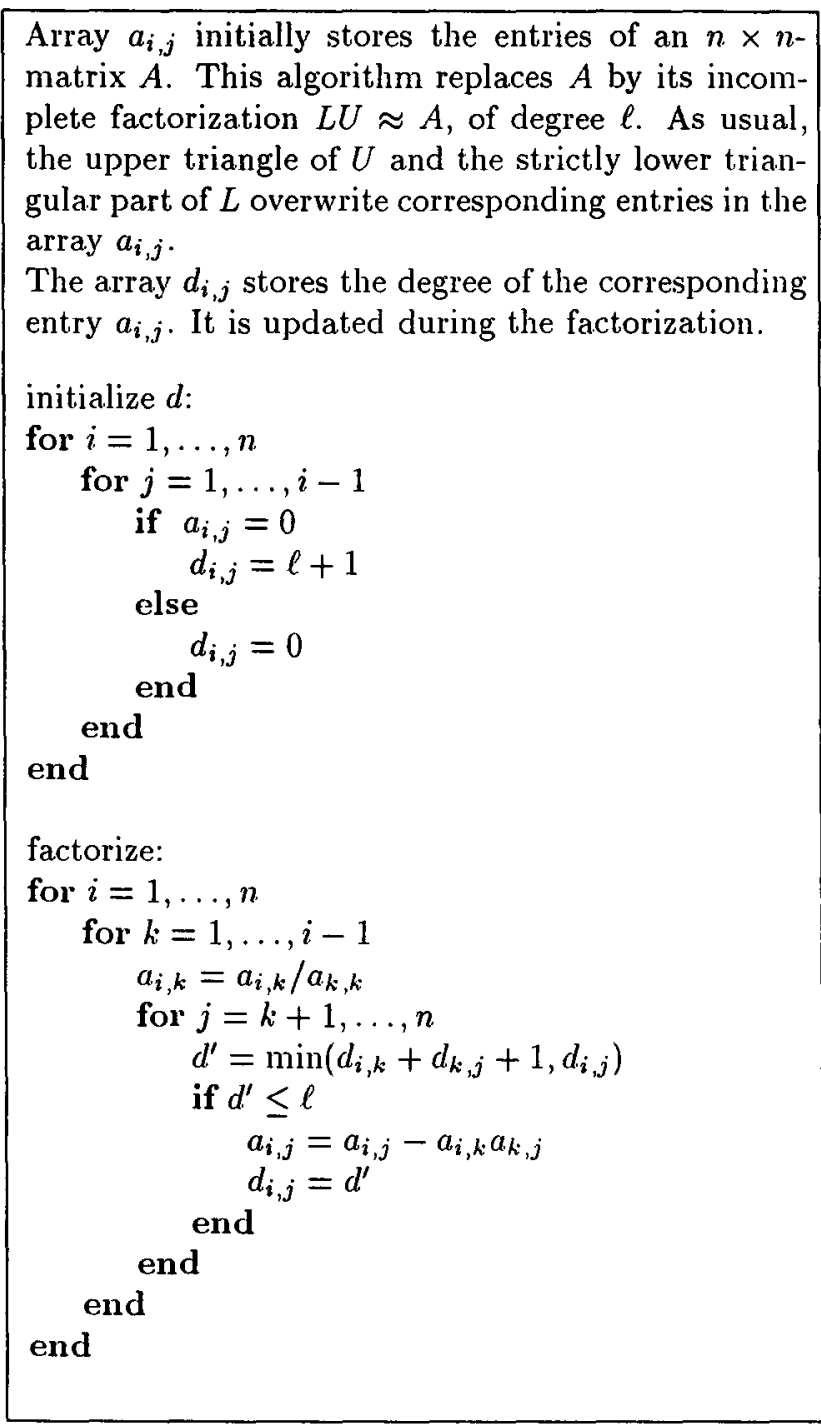

to level $\ell$ is denoted by $\mathrm{I} L U(\ell)$, the incomplete $L U$ factorization of degree $\ell$. Algorithms 1 and 2 specify two slightly different variants of $\operatorname{ILU}(\ell)$ for an $n \times n$ matrix $A$.

In practice, an implementation of Algorithms 1 and 2 would store the arrays $\left(a_{i, j}\right)$ and $\left(d_{i, j}\right)$ in an appropriate data structure and the looping would take on a very special appearance. We discuss details of implementation in a later section.

The nomenclature of incomplete factorizations is not at all standardized. Watts [15] introduced for banded matrices the concept of order, which Behie and Forsyth [4] call degree, to distinguish it from Gustafsson's [9] "first order factorization." Nghiem and Rozon [11] generalize for non-banded matrices. Our definition follows essentially these authors; the difference is that what we call $I L U(\ell)$ would be an incomplete $L U$-factorization of $(\ell+1)$ th degree in their terminol-
AlgorithM 2 The incomplete factorization $\mathrm{I} L U(\ell)$, where the numerical factorization is preceded by a symbolic factorization.

Like Algorithm 1, this algorithm replaces $A$ by its incomplete factorization $L U \approx A$, of degree $\ell$.

The array $d_{i, j}$ is computed by a symbolic factorization before the numerical factorization.

symbolic factorization:

for $i=1, \ldots, n$

for $j=1, \ldots, i-1$

if $a_{i, j}=0$

else

$d_{i, j}=\ell+1$

end

$$
d_{i, j}=0
$$

end

for $k=1, \ldots, i-1$

$$
\text { for } \begin{aligned}
j & =k+1, \ldots, n \\
d_{i, j} & =\min \left(d_{i, k}+d_{k, j}+1, d_{i, j}\right)
\end{aligned}
$$

end

end

numerical factorization:

for $i=1, \ldots, n$

for $k=1, \ldots, i-1$

$a_{i, k}=a_{i, k} / a_{k, k}$

for $j=k+1, \ldots, n$

if $d_{i, j} \leq \ell$

end

$a_{i, j}=a_{i, j}-a_{i, k} a_{k, j}$

end

end

end

ogy. Our use of $\mathrm{I} L U(0)$ conforms to a standard reference [3]. On the other hand, this source defines higher levels of fill differently. In Algorithm 2 the symbolic factorization computes the degree by

$$
d_{i, j}=\min \left(d_{i, k}+d_{k, j}+1, d_{i, j}\right)
$$

According to [3], this line would be replaced by

$$
d_{i, j}=\min \left(\max \left(d_{i, k}, d_{k, j}\right)+1, d_{i, j}\right)
$$

For matrices from five-point discretization on an $I \times J$ grid with natural ordering, both definitions (1) and (2) are equivalent. For three-dimensional or irregular grids, in general, they are not.

The definition in equation (1) harmonizes with the following observation. Let $A$ be a banded matrix with unit main diagonal, at most $k$ nonzero off-diagonal entries per row, all equal $\epsilon$. When $A$ is strongly diagonally dominant, $k \epsilon \ll 1$, then an inductive argument 
easily proves that fill entries of degree $\ell$ (in the sense of equation (1)) are of order $O\left(\epsilon^{\ell+1}\right)$.

Symbolic and Numerical factorization. Algorithm 2 computes the degree of all entries separately, by a symbolic factorization, before the numerical factorization starts. Algorithm 1 updates the degree in the course of numerical factorization. This algorithm may discard a fill term of degree $>\ell$, but later, at the same position in the matrix, retain another fill term of degree $\leq \ell$. By contrast, Algorithm 2 will keep both fill terms. In the end, both algorithms produce the same number of fill entries, but this minor difference in the formulation causes slightly different convergence rates.

A simple example is the $6 \times 6$-matrix

$$
A=\left(\begin{array}{cccccc}
4 & -1 & -1 & 0 & -1 & 0 \\
-1 & 3 & -1 & 0 & 0 & -1 \\
-1 & -1 & 2 & 0 & 0 & 0 \\
0 & 0 & 0 & 2 & -1 & -1 \\
-1 & 0 & 0 & -1 & 2 & 0 \\
0 & -1 & 0 & -1 & 0 & 2
\end{array}\right)
$$

For $\mathrm{I} L U(1)$, Algorithm 2 obtains a complete factorization while Algorithm 1 discards some fill. For this reason, the error matrix $E=L U-A$ has two nonzero entries, $e_{5,6}=e_{6,5}=-3 / 13$.

This distinction is seldom noticed. Besides, for incomplete factorizations of low degree, there is no difference at all. A tedious but straightforward analysis proves the following facts.

Observation. For $\mathrm{I} L U(0)$, the Algorithms 1 and 2 are equivalent. For a five-point stencil on an $I \times J$ grid with natural ordering, the algorithms differ if and only if $\ell \geq 3$. For a seven-point stencil on an $I \times J \times K$ grid with natural ordering, they differ if and only if $\ell \geq$ 2. An alike statement holds for the diagonal ordering D2 [12].

Another example are two-colorable grids. These are grids where the nodes can be colored with two colors so that no two adjacent nodes have the same color. Cartesian grids with five-point or seven-point stencils, for example, are two-colorable. Consider wavefront ordering, which is a generalization of diagonal ordering for arbitrary grids. We define it recursively in the following way: Front 0 is empty. Front 1 contains one node of the grid. Front $k+1$ consists of all nodes that are not in front $k-1$ and that are neighbors of nodes in front $k$.

Observation. For a two-colorable grid with wavefront ordering, Algorithms 1 and 2 produce the same factorization for $\ell<2$. This statement includes, for example, D2 ordering on a three-dimensional cartesian grid.

Proof. An inductive argument for $\ell=0,1,2, \ldots$ establishes that for both variants of $\operatorname{ILU}(\ell)$, fill terms of odd degree couple nodes in the same wavefront only; and fill terms of even degree couple nodes in adjacent wavefronts. It is therefore not possible that Algorithm 1 assigns first to a matrix entry the degree $k$, and later updates it to $k-1$. Specifically, for $\ell=1$, adjacent wavefronts are coupled by entries of degree 0 ; within a wavefront, nodes are coupled by entries of degree 1 .

To sum up, when several matrices with the same sparsity pattern have to be factorized, it is obviously better to compute the symbolic factorization in Algorithm 2 only once. On the other hand, if the structure of the linear system changes with every time step and every outer iteration in a simulator, a new symbolic factorization is necessary at every step. Then Algorithm 1 is more efficient, since it accesses less data without changing the result significantly.

TABLE 1: Amount of fill for an $I \times J \times K$ grid, natural ordering

\begin{tabular}{|c|r|r|r|r|r|r|}
\hline Degree: & \multicolumn{1}{c}{0} & \multicolumn{1}{c}{1} & \multicolumn{1}{c}{2} & \multicolumn{1}{c|}{3} & \multicolumn{1}{c|}{4} & \multicolumn{1}{c|}{5} \\
\hline$I J K$ & 7 & 6 & 10 & 20 & 28 & 36 \\
$I J$ & -2 & -4 & -8 & -16 & -24 & -32 \\
$I K$ & -2 & -4 & -10 & -26 & -50 & -82 \\
$J K$ & -2 & -4 & -12 & -30 & -54 & -86 \\
$I$ & 0 & 2 & 8 & 22 & 46 & 78 \\
$J$ & 0 & 2 & 8 & 20 & 40 & 68 \\
$K$ & 0 & 2 & 8 & 24 & 60 & 124 \\
1 & 0 & 0 & -4 & -14 & -46 & -106 \\
\hline
\end{tabular}

Amount of Fill, Ordering. Without any information about the sparsity pattern of a matrix, there is no way to guess how much fill an $I L U(\ell)$ factorization will produce. As a guideline, the fill in regular grids, with both natural and diagonal ordering, may be used for a rough estimate.

For a seven-point stencil on an $I \times J \times K$ grid that is ordered naturally first in direction $I$, then $J$, then $K$, Table 1 gives the exact amount of fill produced by Algorithms 1 and 2 on various levels. The entries in this table mean that, for example, there are

$$
\begin{gathered}
10 I J K-8 I J-10 I K-12 J K+ \\
8(I+J+K)-4
\end{gathered}
$$

entries of degree 2. The total number of nonzeros in the factorization $I L U(\ell)$ is the sum of these terms from degree 1 to $\ell$. The formulas are valid for $I \geq \max (2, \ell)$ and $J, K \geq 1$.

One conclusion to be drawn from this table is that the amount of fill for higher degrees quickly reaches the boundaries of available memory. Considering the leading terms only, we see: besides the seven bands originally present in a three-dimensional system, there are $6+10+20+28$ bands for a factorization $\mathrm{I} L U(4)$. 
The two-dimensional case follows for $K=1$. Here, fill of higher degree does not grow as fast as in three dimensions. Apart from the five bands originally present in a three-dimensional system, there are $2+2+4+4$ bands for a factorization $I L U(4)$. Each additional degree adds four bands.

A grid with the third dimension much smaller than the number of blocks in $x$ - and $y$-direction behaves more like a small stack of two-dimensional grids. Here too, the demands on memory are not as high as in a "genuinely" three-dimensional case, since negative coefficients of $I J, I K$ and $J K$ are subtracted from the leading term $I J K$.

For diagonal ordering D2 [12], Table 2 gives analogous results. For degree $\ell<3$, they are identical with the values from natural ordering and therefore not repeated here. Note that the leading terms do not grow as rapidly as for natural ordering. This is a strong argument in favor of D2 ordering.

TABLE 2: Amount of fill for an $I \times J \times K$ grid, D2 ordering

\begin{tabular}{|c|r|r|r|}
\hline Degree: & \multicolumn{1}{c|}{3} & \multicolumn{1}{c|}{4} & \multicolumn{1}{c|}{5} \\
\hline$I J K$ & 12 & 16 & 18 \\
$I J$ & -16 & -24 & -36 \\
$I K$ & -16 & -26 & -36 \\
$J K$ & -16 & -30 & -36 \\
$I$ & 18 & 38 & 62 \\
$J$ & 18 & 38 & 62 \\
$K$ & 18 & 38 & 62 \\
1 & -12 & -38 & -72 \\
\hline
\end{tabular}

Sparse Storage Format. An implementation of Algorithm 1 or 2 makes sense only with a sparse storage format for arrays $\left(a_{i, j}\right)$ and $\left(d_{i, j}\right)$. The most timeconsuming tasks then are the computation of the degrees, and the insertion of fill entries in the sparse data structure.

The ordering of the loops in the factorization process in Algorithms 1 and 2 -outermost $i$, then $k$, imnermost $j$-differs from that in the classical formulation of Gaussian elimination. In the terminology of Dongarra, Gustavson and Karp [7], it corresponds to the $i k j j$ variant of Gaussian elimination, while the form most often taught students in a first course in linear algebra is the form $k i . j$. While both formulations compute the same (complete or incomplete) factorization, their patterns of data access differ radically.

In a sparse storage scheme it is usually easier to access data than to insert fill terms into the scheme. We use compressed row storage, a usual scheme for irregular sparsity patterns $[3,6]$. In this format it is easy to access entries row-wise; but it is prohibitively expensive to loop through by columns. Therefore, the form $i k j$ is suitable, since it transforms one row $i$ at a time, by adding scalar multiples of all previous rows $k=1, \ldots, i-1$. The "classical" form $k i j$ would loop through all nonzero entries $a_{i, k}$ in column $k$ for $i=k+1, \ldots, n$; and it would modify the sparsity pattern in the lower right block $\left(a_{i, j}\right), k<i, j$

eqn. Both tasks are extremely inefficient in compressed row storage.

\section{IMPLEMENTING ALGORITHM 1}

Initially, $A$ is stored in compressed row format. The factorization does not overwrite $A$ (the iterative solver needs both the original matrix and its incomplete factorization).

The factors $L$ and $U$ are constructed row by row and are stored in compressed row format. Initially, the data structure reserved for $L$ and $U$ is empty; a vector xhelp of length $n$. contains zeros. Our implementation is laid out in Algorithm 3.

Algorithm 3 Implementation of Algorithm 1 with sparse data in compressed row format.

A simplified outline of sparse incomplete factoriza-
tion; the steps for calculating and updating the de-
gree have been omitted.
for all rows $i=1, \ldots, n$
Block 1
while end of list pointlow not reached
Block 2
for all entries in compressed row $k$ of $U$
Block 3
end
end
Block 4
end

Block 1. Scatter the nonzero entries of row $i$ in $A$ from compressed storage to xhelp.

During scattering, count nonzero entries to the left of the main diagonal and build an ordered list pointlow of column pointers, so that pointlow(k) points to the position of the $k$-th nonzero entry in xhelp before the main diagonal.

Count nonzero entries to the right of the main diagonal and build a list pointup of column pointers, so that pointup $(k)$ points to the position of a nonzero entry in xhelp behind the main diagonal.

Block 2. Advance to next pointer in pointlu and get the corresponding entry in xhelp. This would be entry $a_{i, k}$ in the loop for $k$ in Algorithm 1. Fetch the nonzero entries of row $k$ in $\mathrm{U}$. Calculate pivot piv and store it on $x(k)$. 
As we construct $U$, we order nonzero entries in a row with respect to their degree first, and then with respect to column number. Therefore we do not need to store the degrees of each individual entry in $U$; we only need pointers to the first entry of degree $0,1, \ldots, \ell$ for each row.

Block 3. The innermost loop adds a scalar multiple of row $k$ of $U$ to the vector xhelp: Get next entry $\mathbf{u j}$ and its column index $j$. When the degree of the fill does not exceed $\ell$, update

$$
\operatorname{xhelp}(j)=\operatorname{xhelp}(j)-p i v * u j
$$

The difficult part here is monitoring the degree. A rather awkward nested if-structure controls the various cases (simple update; update and change of degree; insertion of new entry). For any new entry, a pointer is either inserted at the correct position in the ordered list pointlow or appended to the list pointhigh.

Block 4. When elimination in row $i$ is completed, the information in the lists pointlow and ponthigh is used to gather the nonzero entries from xhelp while they are reset to zero in xhelp. A new row is added to the compressed storage of $L$ and $U$.

Besides the arrays for compressed storage of $A, L$ and $U$, this implementation needs $\ell+4$ arrays of length $n$. When the matrix has a maximum bandwidth $\ll n$, these arrays could be shorter, $\ell+6$ times the maximum half-bandwidth.

By careful use of pointers, we have strictly avoided any loop that explicitly scans sparse arrays for nonzero entries. Most of the computational work is strictly proportional to the number of nonzero entries in $A$, except one step in Block 3: The array pointlow is an ordered list. Inserting a new element needs an order of $\log k$ comparisons, when $k$ is the actual length of pointlow. This length is always less than the maximum number of nonzero entries per row in the factor $L$. Nevertheless, this insertion is a costly operation.

\section{SEVERAL UNKNOWNS PER BLOCK}

The algorithms described so far are sometimes called pointwise factorizations to distinguish them from block methods of the type investigated by Concus et al. [5] or Axelsson et al. [2]. There, the blocks result from grouping gridpoints of one row or plane. When the linear system involves more than one variable per gridpoint, blocks of another type occur.

The unknowns and corresponding balance equations may be grouped with respect to their type. For example, take all pressure unknowns first, then water sa.turation, then oil saturation. When there are $q$ types of variables and, correspondingly, $q$ types of equations, the matrix is partitioned into $q \times q$ blocks. The Block in position $(i, j)$ contains the terms that couple unknowns in balance equations of type $i$ to unknowns of type $j$.

More customary is an ordering that for each gridpoint groups the corresponding unknowns. The sparsity pattern of the associated matrix is related to the structure of an IMPES matrix in the following way. An entry $a_{i, j}$ is replaced by an $r \times s$ submatrix that contains for the $r$ equations at gridpoint $i$ their derivatives with respect to the $s$ variables at gridpoint $j$.

Submatrices. A straightforward implementation of I $L U$-factorization for blocked matrices replaces in the pointwise algorithm any operations on matrix entries $a_{i, j}$ by the appropriate operations with $r \times s$ submatrices. Object-oriented programming languages can handle this task elegantly. This fact notwithstanding, the approach is inefficient. In an adaptive-implicit black-oil model, for example, the innermost loops will involve multiplication of $3 \times 3-, 3 \times 1,1 \times 3$ - and $1 \times 1$ matrices in all possible combinations. These are short inner loops, which degrade the performance of any algorithm.

Furthermore, even in a fully implicit formulation many $3 \times 3$-matrices are not dense; nor is the fill caused by them. Treating them as full blocks increases considerably the amount of memory.

Strictly Pointwise Approach. Another way of implementation is simply to ignore the block structure. For Algorithm 1, witch can deal with any sparsity pattern, it does not make any difference whether the matrix results from an IMPES, fully implicit or even more elaborate model. The simulator SURE has employed this approach successfully for several years, where it worked as a robust and reliable solver.

Nevertheless, an obvious drawback of this approach is that it cannot recycle the information from one symbolic factorization for the decomposition of the next matrix (except in the rare instance when the number of variables does not change in any block).

Blockwise Approach, Revisited. In a simulator that changes its model adaptively, the solver at each call encounters linear systems with different sparsity pattern. However, the structure of the linear system does not change irregularly at every step. As Figure 1 shows, there may be a different number of equations per gridblock at each time step, but the grid itself will not change.

We have investigated the following idea. A symbolic factorization as in Algorithm 2 for a matrix with one unknown per gridpoint is performed once; the positions and degrees of nonzero entries are stored in compressed row format. When the actual number and type 
of unknowns is known, it is easy to prepare in advance the appropriate compressed row format for $L$ and $U$. This involves just scanning rowwise through the array that holds the nonzeros from symbolic factorization; each row is "inflated" according to the actual number of variables per block, and the appropriate pointers are stored in the compressed row format for $L$ and $U$. Simultaneously, another important idea can be implemented. While preparing the data structure, it is possible to keep different degrees of fill for different types of unknowns. For saturation unknowns, we do not allow any fill outside the block; and we keep fill from pressure unknowns up to degree $\ell$ as usual. This resembles to some extent an incomplete version of the "combinative" method of Behie and Forsyth [4].

The simulator preprocesses the equations before it sends them to the solver. It already eliminates unknowns that couple only to other unknowns in the same block; and it interchanges equations according to a pivoting strategy. Proper scaling and pivoting improves the efficacy of the preconditioner.

When the column pointers for nonzero entries $L$ and $U$ in compressed storage format are prepared in advance, the numerical factorization in Algorithm 2 works efficiently. Neither does it need nested ifstructures nor bookkeeping in ordered lists. Thus, we apply the algorithm for numerical factorization in pointwise form for a sparsity pattern that has been prepared based on the blockwise symbolic factorization.

Algorithm 4 presents the structure of an iterative solver based on those ideas.

Algorithm 4 Structure of an iterative solver for an adaptive-implicit formulation of flow equations on an irregular grid.

Each call to the solver executes these steps
if first call, or changed degree $\ell$
symbolic factorization;
end
pivoting and partial elimination within blocks;
prepare data structure for $L$ and $U ;$
numerical factorization;
preconditioned ORTHOMIN iterations;
if convergence failed
and degree $\ell<\ell_{\text {max }}$
$\quad$ increase degree and restart solver;
else if convergence fajled
and degree $\ell=\ell_{\text {max }}$
$\quad$ cut timestep and restart outer iterations;
else if fast convergence
and degree $\ell>\ell_{\text {min }}$
decrease degree;
end

Practical Experiences. We have compared in the reservoir simulator SURE a strictly pointwise approach, Algorithm 3, with the blockwise approach as outlined in Algorithm 4. Table 3 illustrates a typical case, a history matching (1963-1995) of a field for underground gas storage. It is representative for a multitude of other simulations. We chose this particular case because it was the first full-field simulation attempted with our version of blockwise factorization. The model was a dynamically implicit formulation for 9483 blocks. Time was measured by the profiling utility prof under Unix on an IBM workstation. There were 571 calls to the solver (blockwise), and 978 solver calls (pointwise). This difference stems from different time steps and a different number of outer iterations. Since the simulator gets feedback about the convergence rates in the solver, it adjusts time steps accordingly. The degree $\ell$ in the incomplete factorizations varied between $3 \leq \ell \leq 6$.

Several factors contribute to the increase of computational speed in this example. First, the I $L U$ factorization is much more efficient in the blockwise approach. Second, the selective discarding of fill terms that correspond to saturations reduces the number of entries in $L$ and $U$. Consequently, the application of the preconditioner within ORTHOMIN takes less time. Third, and surprisingly, it turned out that discarding saturation terms and keeping only pressurerelated fill up to degree $\ell$ in some cases even improved the convergence of the linear iterations; in many other cases it did at least no harm. The average number of ORTHOMIN iterations dropped from 75.3 (pointwise) to 46.0 (blockwise). Fourth, the routine that controls the time-step length profits from the increased robustness. Whenever a time step has to be cut and repeated because the solver failed to converge, this is a disaster in terms of performance. A routine that carefully selects the timestep within the solver's capabilities is extremely important.

\section{CONCLUSIONS}

High-degree incomplete factorizations can be carried out efficiently to solve adaptive-implicit formulations of reservoir equations on irregular grids. Although the sequential structure of $L U$-factorization does not allow straightforward parallelization, these codes are very suitable for workstations and vector computers.

\section{REFERENCES}

[1] J. R. Appleyard and I. M. Cheshire. Nested factorization. Technical report, Paper SPE 12264, Seventh SPE Symposium on Reservoir Simulation, San Francisco, California, 1983. 
TABLE 3: A comparison of total CPU time, blockwise versus pointwise incomplete factorization

\begin{tabular}{|c|rr|rr|}
\cline { 2 - 5 } \multicolumn{1}{c|}{} & Time $(\mathrm{sec})$ & & \multicolumn{2}{c|}{ Time $(\mathrm{sec})$} \\
\hline I $L U$-factorization & 152.35 & $4.1 \%$ & 829.42 & $22.3 \%$ \\
\hline ORTHOMIN iterations & 892.95 & $24.0 \%$ & 2885.15 & $77.7 \%$ \\
\hline solver, total & 1045.30 & $28.1 \%$ & 3714.57 & $100.0 \%$ \\
\hline
\end{tabular}

[2] O. Axelsson, S. Brinkkemper, and V. P. Il'in. On some versions of incomplete block-matrix factorization iterative methods. Lin. Alg. Appl., 58:315,1984 .

[3] R. Barret, M. Berry, T. F. Chan, J. Demmel, J. Donato, J. Dongarra, V. Eijkhout, R. Pozo, C. Romine, and H. van der Vorst. Templates for the Solution of Linear Systems: Building Blocks for Iterative Methods. SIAM, Society for Industrial and Applied Mathematics, Philadelphia, 1994

[4] G. A. Behie and J. P. A. Forsyth. Incomplete factorization methods for fully implicit simulation of enhanced oil recovery. SIAM J. Sci. Stat. Comput., 5(3):543-561, September 1984.

[5] P. Concus, G. H. Golub, and G. Meurant. Block preconditioning for the conjugate gradient method. SIAM J. Sci. Stat. Comput., 6:220-252, 1985.

[6] J. J. Dongarra, I. S. Duff, D. C. Sorensen, and H. A. van der Vorst. Solving Linear Systems on Vector and Shared Memory Computers. Society for Industrial and Applied Mathematics, Philadelphia, 1991.

[7] J. J. Dongarra., F. G. Gustavson, and A. Karp. Implementing linear algebra algorithms for dense matrices on a vector pipeline machine. SIAM Review, 26(1):91-112, January 1984.

[8] G. H. Golub and C. F. V. Loan. Matrix Computations. John Hopkins University Press, Baltimore and London, second edition, 1989.

[9] I. Gustafsson. A class of first order factorization methods. BIT, 18:142-156, 1978.

[10] J. A. Meijerink and H. A. van der Vorst. An iterative solution method for linear systems of whitch the coefficient matrix is a symmetric m-matrix. Math. Comp., 31(137):148-162, 1977.

[11] L. Nghiem and B. Rozon. A unified and flexible approach for handling and solving large systems of equations in reservoir simulation. In Proceedings of the First and Second International Forum. on Reservoir Simulation held at Alpbach, Austria.
P. Steiner, Leoben, Austria, September 1988 and September 1989.

[12] H. S. Price and K. Coats. Direct methods in reservoir simulation. Trans. SPE of AIME, 257:295308,1974 .

[13] G. W. Thomas and D. H. Thurnau. Reservoir simulation using an adaptive implicit method. Soc. Petrol. Eng. J., 23:759-768, October 1983.

[14] P. K. W. Vinsome. ORTHOMIN, an iterative method for solving sparse sets of simultaneous linear equations. In Proceedings of the Fourth SPE Symposium on Reservoir Simulation held in Los Angeles, CA. Society of Petroleum Engineers, February 19-20 1976. Paper SPE 5729.

[15] J. W. Watts. A conjugate gradient-truncated direct method for the iterative solution of the reservoir simulation pressure equation. Soc. Petrol. Eng. J., 21:345-353, 1981. 
